\title{
On the numerical solution of pseudoparabolic equations
}

\author{
Robert P. Gilbert ${ }^{1}$ ) and Leroy R. LUNDiN
}

In der Arbeit werden diskrete Analoga der Fundamentalsingularitäten für die pseudopàrabolische Differentialgleichung gefunden. Dabei dient eine von Coltos und Gilbert für ana. lytische Koeffizienten entwickelte Methode zur Motivation der Annäherung. In dér Tat, die Green'sche Differenzenfunktion spielt hier die gleiche Rolle wie im analytischen Fall. Das Linienverfahren dient zur Behandlung der. Differentiation nach der Zèit. Schließlich werden Fchlerabschätzungen erhalten, in denen die stetigen Lösungen mit den Differenzenapproximationen verglichen werden.

В работе найдены дискретные аналюги фундаментальных сингулярностей для псевдопараболических дифференциальных уравиении. При этом метод, разработанный Cоцток и Gпвевт для аналитических коэффициентов, служит для мотивировки аппрожсимации. Действительно, функция разности Грина играет здесь ту же самую роль как в аналитическом случае. Метод служит для обработки дифференцирования по времени. Наконег получены оценки погрешностей, в корорых сравнинаются непрерывные решения с разностными аппроксимациями.

In this work the authors find discrete analogues of the fundamental singularities for pseudoparabolic equations. The method developed by CoLton and GLBERT for analytic coefficients is used to motivate the approach. Indeed, the finite difference Green's function is seen to play the same role here as in the analytic case. The method of lines is employed to treat the time differentiation. Furthermore, error estimates are obtained which compare the continuous solutions to the finite difference approximations.

\section{Introduction}

The investigation of partial differential equations of pseudo-parabolic type have received much interest recently. In particular, the methods of functional, analysis have been effectively brought to bear on these problems by' SHowalter and TrNG $[9-1.1,13,14]$. An alternate approach, which stresses the use of function theoretic methods has been'developed by Coltow, Gilbert and Hsicio [4, 5, 7, 8]. Indeed, a fairly general function theoretic method now exists for, investigating pseudo-paraboljc equations in two space variables $[7,8]$. These are equations of the form

$$
\mathfrak{Z}[u]:=\underset{\sim}{M}\left[u_{t}\right]-L[u]=0
$$

where ord $\underset{\sim}{M}>$ ord $\underset{\sim}{L}$ and $\underline{M}$ is elliptic.

Pseudobarabolic equations arise in a variety of physical problems, such as the velocity of non-steady flows of viscous fluids [1], and the hydrostatic excess pressure occuring during the consolidation of clay [12].

1) This work was supported in part by the National Science Foundation through Grant No. MCS 78-02452, and the Department of Energy through Grant No. DE-AC01-81 ER-1067. 
The papers cited above by GILBERT [7], and Gilbert-Hsiao [8] generalize the approach used by Coiton $[4,5]$ for the case where ord $M=2$ to order $2 n$. In these works the approach depends on the analytical construction of fundamental singular solutions to the adjoint equation

$$
\underline{Q}^{*}[v]:=\underline{M}^{*}\left[v_{t}\right]+\underset{\sim}{L} *[v]=0,
$$

where $M^{*}$, and $L^{*}$ are the formal Lagrange adjoint operators for $M$ and $L$ respectively. It has been shown, moreover, that it is possible to develop the fundamental singularity in the form

$$
S(P, t ; Q, \tau):=A(P, t ; Q, \tau) \ln \frac{1}{r}+B(P, t ; Q, \tau)
$$

where

, and

$$
r:=|P-Q|, \quad P:=(x, y), \quad Q:=(\xi, \eta),
$$

$$
\begin{aligned}
& A(P, t ; Q, \tau):=\sum_{j=1}^{\infty} A_{j}(P, Q) \frac{(t-\tau)^{j}}{j !} \\
& B(P, t ; Q, \tau):=\sum_{j=1}^{\infty} B_{j}(P, Q) \frac{(t-\tau)^{j}}{j !}
\end{aligned}
$$

The remarkable result of this approach is that $A(P, Q)$ is the Riemann function associated with the operator $M$, that is, if $M_{1}$ is written as a hyperbolic operator by formally mapping $(x, y) \rightarrow\left(z, z^{*}\right), z=\dot{x}+\widetilde{*} y, z^{*}=x-i y$. The other coefficients $A_{j}(j \geqq 2)$, and the $B_{j}(j \geqq 1)$ may then be obtained by recursive schemes.

- Two obvious disadvantages of the above method are that (1) the coefficients of $\underset{\widetilde{M}}{M}$ and $L$ must be analytic in the space variables, and $(2)$ it is very difficult, in general, to do the necessary analytical computations for the $A_{j}(j \geqq 2), B_{j}((\geqq 1)$ even when the Riemann function for $M$ is already known.

It is the purpose of the present paper to circumyent these difficulties by replacing the required analytical computations by numerical algorithms. Furthermore, we modify the approach cited above to include the case of nonanalytic coefficients. This permits circumvention of the necessary procedure of analytically continuing the coefficients into the $\left(z, z^{*}\right)$ space. For simplicity of exposition we discuss only the case where

$$
\underline{M}[v]:=\triangle v-q(P) v, \quad q(P)>0 \text { for } P \in \Omega,
$$

and

$$
L[v]:=a(P) v, \quad a(P)<0 \text { for } P \in \Omega,
$$

and where for purposes of numerical estimation we assume that the coefficients are in $C^{2, \alpha}(\bar{\Omega})$, [3], and in particular we consider the initial-boundary value problem

$$
\begin{aligned}
& \mathscr{L}[u](P, t)=F(P, t), \quad(P, t) \in \Omega \times[0, \infty) \\
& u(P, t)=f(P, t) \quad \text { for } \quad P_{1} \in C:=\dot{\Omega}, \quad t>0 \\
& u(P, 0)=0, \quad P \in \bar{\Omega} .
\end{aligned}
$$

Here $\Omega$ is taken to be a simply-connected region, such that the boundary $\dot{\Omega}$, is smooth enough for the various Green's identities to hold.

In the exposition which follows we shall treat first the problem of (1.4) with continuous coefficients, and devèlop a representation formula for its solution. Having done this we shall turn our attention to various discretized forms of this problem and obtain error estimates conparing the solution of (1.4) with the discretized solutions. 


\section{The Continuous Case}

Following the idea of $[4,5]$ and $[7,8]$ we attempt to construct a fundamental solution. By.a "Green's function". for (1.4), we shall mean a function $G$ defined on $\bar{\Omega} \times \bar{\Omega} \times[0, \infty)$ which satisfies

$$
\left.\begin{array}{lll}
\mathfrak{Q}_{P} G(P, Q ; t)=\delta(P-Q) & \text { for } P \in \Omega, & i \geqq 0 ; \\
G(P, Q ; t)=0 & \text { for } P \in C, \quad t>0 ; \\
G(P, Q ; 0)=\ddot{0} & \text { for } P, Q \in \bar{\Omega} .
\end{array}\right\}
$$

Theorem 2.1: There is a unique function $G$ which is analytic in $t$ and satisfies (2:1). IF urthermore $G$ may be represented in the form

$$
\text { - } G(P, Q ; t)=\sum_{n=0}^{\infty} G_{n}(P, Q) \frac{t^{n+1}}{(n+1) !}
$$

where $G_{0}^{\prime}$ is the Grèen's function associated with the problem $\underset{\sim}{M} w=f,\left.w\right|_{c}=0$. The coefficients $G_{n+1}$ may be uniquely determined as solutions of the recursive system

$$
\left.\begin{array}{lll}
M_{P} G_{n+1}(P, Q)=L_{P} G_{n}(P, Q) & \text { for } & P \in \Omega, \\
G_{n+1}(P, Q)=0 & \text { for } & P \in C .
\end{array}\right\}
$$

Proof: For the general scheme used to construct fundamental solutions see $[\dot{4}, 5$, 7, 8]. The result then follows by noting $G$ satisfies definition (2.1). It can be seen that $G$ converges as it is a special fundamental solution of the form investigated already in $[4,5,7,8]$

In what follows, $G$ will always be taken to mean the fundamental solution (2.2-2.3). An important property of Green's functions for elliptic equations is their reproducing property. This is also the case for our $G$. Let $v$ be defined on $\bar{\Omega} \times[0, \infty)$ such that it is simultaneously $C^{2}$ in the space-variables and $C^{1}$ in the time variable. We designate this space as $\sum$. Starting with the integral identity

$$
\begin{aligned}
v(P, t)= & \int_{0}^{t} d \tau \int_{Q} \frac{\partial}{\partial \tau} v(Q, \tau) \Omega_{Q}[\dot{G}(Q, P ; t-\tau)] d A\left(\Omega_{Q}\right) \\
= & -\int_{0}^{t} d \tau \int_{Q} \frac{\partial}{\partial \tau} v(Q, i)\left\{[\triangle Q-q(Q)] \frac{\partial}{\partial \tau}[G(Q, P ; t-\tau)]\right. \\
& +a(Q) G(Q, P ; t-\tau)\} d A\left(\Omega_{Q}^{\prime}\right), \quad d A:=d x \cdot d y,
\end{aligned}
$$

and applying Green's third identity one obtains

$$
\begin{aligned}
v(P, t)= & -\int_{0}^{t} d \tau \int_{0}\left\{M \cdot\left[\frac{\partial}{\partial \tau} v\right](Q, \tau): \frac{\partial}{\partial \tau}[G(Q, P ; t-\tau)]\right. \\
& \left.+a(Q) \frac{\partial}{\partial \tau} v(Q, \tau) G(Q, P ; t-\tau)\right\} d A\left(\Omega_{Q}\right) \\
& -\int_{0}^{\ell} d \tau \int_{c} \frac{\partial}{\partial \tau} v(Q, \tau) \frac{\partial^{2}}{\partial n_{Q} \partial \tau}[G(Q, P ; t-\tau)] d \sigma_{Q}
\end{aligned}
$$


Here $\frac{\partial}{\partial n_{Q}}$ denótes differentiation in the direction of the outward normal, and $d \sigma_{Q}$ denotes the arc length differential along $C$. Integrating by parts the $a(Q)$ term and using the initial conditions for $v$ and $G$, yields

$$
\begin{aligned}
& \int_{0}^{t} d \tau \int_{Q} a(Q) \frac{\partial}{\partial \tau} v(Q, \tau) G(Q, P ; t-\tau) d A\left(\Omega_{Q}\right) \\
& =-\int_{0}^{t} a(Q) v(Q, \tau) \frac{\partial}{\partial \tau}[G(Q, P ; t-\tau)] d A\left(\Omega_{Q}\right) .
\end{aligned}
$$

We obtain in this way the integral representation given in the following theorem.

Theorem 2.2: Let $v \in \sum$ and satisfy the homogeneous iñitial condition $v(P, 0)=0$ for $P \in \Omega$. Then for $(P, t) \in \bar{\Omega} \times[0, t)$, v has the representation

$$
\begin{aligned}
v(P, t)= & -\int_{0}^{t} d \tau \int_{\Omega} \frac{\partial}{\partial \tau} G(Q, P ; t-\tau) \Omega v(Q, \tau) d A\left(\Omega_{Q}\right) \\
& -\int_{0}^{t} d \tau \int_{C} \frac{\partial}{\partial \tau} v(Q, \tau) \frac{\partial}{\partial n_{Q}} \frac{\partial}{\partial \tau} G(Q, P ; t-\tau) d \sigma_{Q} .
\end{aligned}
$$

\section{Diskretization in the Space Variables}

A natural method of approximate the fundamental solution $G(P, Q ; t)$ of $(2.2)$ is to approximate the coefficients of the powers of $t$. For simplicity in exposition we first assume that $\Omega$ is a rectangle such that we may place a rectangular grid of equal spacing over $\Omega$ and thereby discretize the space variables. In the usual way we designate certain grid points as interior points and their set as. $\Omega_{h}$. The boundary points are the intersections of the mesh with $C$, and this set is designated by $C_{h}$. We set $\bar{\Omega}_{h}:=\Omega_{h} \cup C_{n}$. Our particular choice for $\Omega$ does not require that the neighborhood of the boundary be treated in a special way at this point. The discrete version of $\mathcal{Q}$ is obtained by replacing $\Delta$ by its centered-difference approximation $\Delta_{h}$. This results in the problem

$$
\left.\begin{array}{ll}
\mathfrak{Q}_{h} u^{h}(P, t)=F(P, t) & \text { for } \quad(P, t) \in \Omega_{h} \times[0, \infty) \\
u^{h}(P, t)=f(P, t) & \text { for } P \in C_{h}, \quad t>0, \\
u^{h}(P, 0)=0, & \text { for } \quad P \in \bar{\Omega}_{h},
\end{array}\right\}
$$

where $\mathfrak{L}_{h} v:=M_{h} \hat{v}_{t}-L v, M_{h}:=\triangle_{h}-q E$, and where $u_{1}^{h}$ is defined on $\Omega_{h} \times[0, \infty)$ and is analytic in its second variable.

As a space discretized-Green's function we shall require a function $G^{\text {h }}$ defined on $\bar{\Omega}_{h} \times \bar{\Omega}_{h} \times[0, \infty)$ which is analytic in $t$ and satisfies

$$
\left.\begin{array}{llll}
\mathcal{Q}_{h, P} G^{h}(P, Q ; t)=-h^{-2} \delta(P, Q) & \text { for } & P, Q \in \Omega_{h}, & t \geqq 0 \\
G^{h}(P, Q ; t)=\delta(P, Q) t & \text { for } & P \in C_{h}, & t>0, \\
G^{h}(P, Q ; 0)=0 & \text { for } P, Q \in \bar{\Omega}_{h}, &
\end{array}\right\}
$$


where

$$
\delta(P, Q):=\left\{\begin{array}{lll}
1, & \text { if } & P=Q \\
0 & \text { if } & P \neq 0
\end{array}\right.
$$

For a review of the discrete generalizations of the classical fundamental singular solutions the reader is referred to WENDLAND [16: Chapter 7].

Theorem 3.1: Problems (3.1) and (3.2) have unique solutions, designated by $u^{h}$ and $G^{*}$ respectively. Moreover, they have expansions of the forms

$$
\begin{aligned}
& u^{h}(P, Q ; t)=\sum_{n=0}^{\infty} u_{n}^{h}(P, Q) \frac{t^{n+1}}{(n+1) !} \\
& G^{h}(P, Q ; t)=\sum_{n=0}^{\infty} G_{n}^{n}(P, Q) \frac{t^{n+1}}{(n+1) !}
\end{aligned}
$$

Here $G_{0}{ }^{h}(P, Q)$ is the discrele Green's function associated with $M_{h}$, and the $G_{n+1}^{h}$ are determined recursively.

Proof: Direct substitution of (3.2b) into the differential equation of (3.2) and aomparing powers of $t$ shows that $G_{0}{ }^{h}$ must be the discrete Green's function as defined cbove. Furthermore, we must have fór. $n \geqq 0$ that $G_{n+1}^{h}$ are the unique solutions of

$$
\begin{aligned}
& M_{h, P}\left[G_{n+1}^{h}(P, Q)\right]=L_{P}\left[G_{n}{ }^{h}(P, Q)\right] \quad:\left(P \in \Omega_{h}\right), \quad G_{n+1}^{h}(P, Q)=0 \\
& \text { for } P \in C_{h},
\end{aligned}
$$

which yields

$$
\dot{G}_{n+1}^{h}(P, Q)=-h^{2} \sum_{T \in \Omega_{n}} G_{0}{ }^{h}(P, T) G_{n}{ }^{h}(T, Q) a(T)
$$

To show that the infinite series $(3.2 \mathrm{~b})$ exists it is sufficient to demonstrate that the finite sum of positive terms ${ }^{2}$ )

$$
\sum_{Q \in Q_{n}} G^{n}(P, Q, t) \quad(t>0)
$$

is bounded. To this end, we note that from Lemma 5.2 we have the inequality

$$
\sum_{Q \in \Omega_{A}} G_{0}{ }^{B}(P, Q) \leqq \frac{k}{h^{2}} \quad(k>0)
$$

where $k:=\left[\min _{\dot{Q} \in \Omega_{n}} q(Q)\right]^{-1} \cdot$ Consequently

Hence,

$$
h^{2} \sum_{Q \in \Omega_{h}} h^{2} \sum_{T \in \Omega_{n}} G_{0}{ }^{h}(P, T) \dot{G}_{0}{ }^{h}(T, Q) \leqq k h^{2} \dot{\sum}_{T \in \Omega_{h}} G_{0}{ }^{n}(P, T) \leqq k^{2}
$$

$$
h^{2} \sum_{Q \in D_{n}} h^{2} \sum_{T \in \Omega_{n}} G_{0}^{n}(P, T) G_{n}{ }^{n}(\dot{T}, Q) a(T) \leqq A^{n+1}[k]^{n+2}
$$

where $A \vdots=\max _{T \in O_{n}}|a(T)| \dot{\text { and }} \sum_{Q \in Q_{n}} G^{n}(P, Q, t) \leqq \frac{t}{h^{2}} k \exp [A t k] \quad(h>0)$. Uniqueness of the solution to either problem follows by considering the case of homogeneous data.

2) That each $G_{n}{ }^{h}(P, Q) \geqq 0$ is shown in the proof of Lemma 5.1. 
Existence of the solution (3.2a) follows from its unique representation in terms of the Green's function, which we list below.

Theorem 3.2: Let $v \in \sum$ and satisfy the homogeneous initial data $v^{\text {h }}(P, 0)=0$ for $P . \in \Omega_{h}$. Then for each $P^{\prime} \in \Omega_{h}$,

$$
\begin{aligned}
v(P, t)= & h^{2} \int_{0}^{t} d \tau \sum_{Q \in \Omega_{n}} \frac{d}{d t} G^{n}(P, Q ; t-\tau) \mathfrak{L}_{h} v(Q, \tau) \\
& +\int_{0}^{\ell} d \tau \sum_{Q \in \Omega_{n}} G^{n}(P, Q ; t-\tau) \frac{d v}{d \tau}(Q, \tau) .
\end{aligned}
$$

Proof: Let $w(P, t)$ denote the right-hand side of (3.4). Clearly, $w(P, 0)=0$ for all $P$. If $P \in C_{h}$ and $t>0, \frac{d}{d t} G(P, Q ; t-\tau)=\delta(P, Q)$ and so

$$
\begin{aligned}
w(P, t) & =\int_{0}^{t} d \tau \sum_{Q \in C_{h}} \delta(P, Q) \frac{d}{d \tau} v(Q, \tau)=\int_{0}^{t} \frac{d v}{d \tau}(P, \tau) d \tau \\
& =v(P, t)-v(P, 0)=v(P, t)
\end{aligned}
$$

Integrating (3.4) by parts yields

$$
\begin{aligned}
w(P, t)= & -h^{2} \sum_{Q \in \Omega_{h}} G^{h}(P, Q ; t) \mathfrak{L}_{h} v(Q, 0)-\sum_{Q \in C_{n}} G^{h}(P, Q ; t) \frac{d v}{d t}(Q, 0) \\
& -h^{2} \int_{0}^{t} d \tau \sum_{Q \in \Omega_{h}} G^{h}(P, Q ; t-\tau) \frac{d}{d \tau}\left[\mathfrak{L}_{h} v(Q, \tau)\right] \\
& -\int_{0}^{t} d \tau \sum_{Q \in C_{h}} G^{h}(P, Q ; t-\tau) \frac{d^{2} v}{d \tau^{2}}(Q, \tau)
\end{aligned}
$$

Thus, for $P \dot{\epsilon} \Omega_{h}$ and $t \geqq 0$,

$$
\begin{aligned}
\mathfrak{Q}_{h} w(P, t)= & \sum_{Q \in \Omega_{h}} \delta(P, Q) \cdot \Omega_{h} v(Q, 0)+h^{-2} \sum_{Q \in C_{h}} \delta(P, Q) \frac{d v}{d t}(Q, 0) \\
& +\int_{0}^{t} d \tau \sum_{Q \in \Omega_{h}} \delta(P, Q) \frac{d}{d \tau}\left[\Omega_{h}^{i} v(Q, \tau)\right] \\
& \quad+h^{-2} \int_{0}^{t} d \tau \sum_{Q \in C_{h}} \delta(P, Q) \frac{d^{2} v}{d \tau^{2}}(Q, \tau) \\
& \quad+\int_{0}^{t} \frac{d}{d \tau}\left[\Omega_{h} v(Q ; \tau)\right] d \tau=\mathfrak{Q}_{h} v(P, t) .
\end{aligned}
$$

From the uniqueness result of Theorem 3.1 follows that $w \equiv v$, and so the proof is complete 


\section{The Method of Lines}

One method of removing the infinite sum in the representation of the fundamental singularity is to truncate the series, another and perhaps better for numerical procedures is to discretize the time variable [15]. We do this now, using equal-spacing with step-size $k$, and use a forward-difference scheme for which the discrete operator is

$$
\mathfrak{Q}_{k} F_{i}:=k^{-1} M\left[v_{i+1}-v_{i}\right]-L v_{i} \text {. }
$$

The adjoint backward-difference operator is

$$
\mathfrak{Q}_{k}{ }^{*} \cdot \dot{v}_{i+1}=k^{-1} M\left[v_{i+1}-v_{i}\right]+L v_{i+1} \text {. }
$$

Let $\mathbf{N}$ denote the set of natural numbers and $\mathbf{N}^{+}=\mathbf{N}-\{0\}$. We seek $G^{k}$ defined on $\bar{\Omega} \times \bar{\Omega} \times \mathbf{N}$ satisfying

$$
\left.\begin{array}{lll}
\mathfrak{L}_{k, P}^{F} G^{k}(P, Q ; i)=\delta(P-Q) & \text { for } P \in \Omega, & P \in \mathbf{N} \\
G^{k}(P, Q ; i)=0 & \text { for } P \in C, \quad i \in \mathbf{N}^{+}, \\
G^{k}(P, Q ; 0)=0 & \ddots & \text { for } P, Q \in \Omega .
\end{array}\right\}
$$

Theorem 4.1: $G^{k}$ exists and is unique. Furthermore, $G^{k}(P, Q ; 0) \equiv 0$ and for $i \in \mathbf{N}^{+}$,

$$
G^{k}(P, Q ; i)=\sum_{j=1}^{i}\left(\begin{array}{l}
i \\
j
\end{array}\right) k^{j} G_{j-1}(P, Q)
$$

where $\left\{G_{j}\right\}_{j=0}^{\infty}$ is as in (2.3).

Proof: Existence may be verified directly using formula (4.2) and substituting into" (4.1). Uniqueness may be shown using formula (4.3) which is given below.

Lemma 4.2: Let $i$ be a (fixed) positive integer and let. $Q \in \bar{\Omega}$. Then for $1 \leqq j \leqq i$, $G^{k}(P, Q ; i-j)$ satisfies

$$
\begin{cases}\mathcal{Q}_{k, P}^{*},{ }_{j} G^{k}(P, Q ; i-j)=-\delta(P-Q) & \text { for } P \in \Omega, \\ G^{k}(P, Q ; i-j)=0 & \text { for } P \in C .\end{cases}
$$

Theorem 4.3: Let $\left\{v_{i}\right\}_{i \in \mathrm{N}}$ be defined on $\bar{\Omega}$ with $v_{0} \equiv 0$. Then for each $i \in \mathbf{N}$ and for each $P \in \Omega$ we have the representation formula

$$
\begin{aligned}
v_{i+1}(P)= & \sum_{j=0}^{i} \int_{Q}\left[G^{k}(Q, P ; i-j+1)-G^{k}(Q, P ; i-j)\right] \mathfrak{L}_{k}{ }^{F} v_{j}(Q) d A\left(\Omega_{Q}\right) \\
& +k^{-1} \sum_{j=0}^{i} \int_{C}\left[v_{j+1}(Q)-v_{j}(Q)\right] \frac{\partial}{\partial n_{Q}}\left[G^{k}(Q, P ; i-j+1)\right. \\
& \left.-\dot{G}^{k}(Q, P ; i-j)\right] \dot{d} \sigma_{Q}
\end{aligned}
$$

Proof: We begin by employing Lemma 4.2, a telescoping series and the definition of $v_{0}$. Setting $\Gamma_{j}(P, Q)=G^{k}(P, Q ; i+1-j)$ we have

$$
\begin{aligned}
v_{i+1}(P)= & -\sum_{j=0}^{i} \int_{\Omega}\left[v_{j+1}(Q)-v_{j}(Q)\right] \Omega_{k, Q}^{* B} \Gamma_{j+1}(Q, P) d A\left(\Omega_{Q}\right) \\
= & -\sum_{j=0}^{i} \int_{\Omega}\left[v_{j+1}(Q)-v_{j}(Q)\right]\left\{k^{-1} M_{Q}\left[\Gamma_{j+1}(Q, P)-\Gamma_{j}(Q, P)\right]\right. \\
& \left.+a(Q) \Gamma_{j+1}(Q, P)\right\} d A\left(\Omega_{Q}\right)
\end{aligned}
$$




$$
\begin{aligned}
= & -\sum_{j=0}^{i} \int_{\Omega}\left[\Gamma_{i+1}(Q, P)-\Gamma_{j}(Q, P)\right] \mathfrak{I}_{k} v_{j}(Q) d a\left(\Omega_{Q}\right) \\
& -k^{-1} \sum_{j=0}^{i} \int_{C}\left[v_{j+1}(Q)-v_{j}(Q)\right] \frac{\partial}{\partial n_{Q}}\left[\Gamma_{j+1}(Q, P)-\Gamma_{j}(Q, P)\right] d \sigma_{0} \\
& -\sum_{j=1}^{i} \int_{\Omega}\left\{a(Q) v_{j}(Q)\left[\Gamma_{j+1}(Q, P)-\Gamma_{j}(Q, P)\right]\right. \\
& \left.+a(Q) \Gamma_{j+1}(Q, P)\left[v_{j+1}(Q)-v_{j}(Q)\right]\right\} d A\left(\Omega_{0}\right)
\end{aligned}
$$

The last sum vanishes due to obvious cancellations; hence, the proof is complete upon replacing the $\Gamma_{j}$-terms by the corresponding $G^{k}$-terms

At this point we investigate the simultaneous discretization of the space and time variables. To this end wë define $\bar{\Omega}_{h}:=\Omega_{h} \cup C_{h}$ as in Section 3 , and we consider the operator

$$
\mathcal{Q}_{k, h}^{F} v_{i}^{h}(P):=M_{h}\left[v_{i+l}^{h}(P)-\dot{v}_{i}^{h}(P)\right]-\operatorname{Lv}_{i}^{h}(P)
$$

where $\left\{v_{i}^{h}\right\}_{i \in \mathbb{N}}$ is a sequence of functions defined on $\bar{\Omega}_{h}$. We scek $\left\{u_{i}^{k, h}\right\}_{i \in \mathrm{N}}$ satisfying

$$
\left.\begin{array}{lll}
\mathfrak{L}_{k, h}^{F} u_{i}^{k, h}(P)=F(P, i k) & \text { for } P \in \Omega_{h}, & i \in \mathbf{N}, \\
u_{i}^{k, h}(P)=f(P ; i k) & \text { for } \quad P \in C_{h}, & i \in \mathbf{N}^{+}, \\
u_{0}^{k, h}(P)=0 \quad 1 & \text { for } \quad P \in \Omega_{h} . & .
\end{array}\right\}
$$

Theorem 4.4: There exists a unique solution $\left\{u_{i}^{k, n}\right\}_{i \in \mathrm{N}}$ to problem (4.4).

Proof: This follows by the fact that for each fixed $i$ we may uniquely solve $M_{h}\left[u_{i+1}^{h}(P)\right]=F(P, i \dot{k})+M_{h}\left[u_{i}^{h}(P)\right]-L u_{i}^{h}(P)$ for $u_{i+1}^{k, h}$ in terms of a known $u_{i}^{h}$. Indeed

$$
\begin{aligned}
u_{i+1}^{k, h}(P)= & -h^{2} \cdot \sum_{Q \in O_{n}} G_{0}{ }^{h}(P, Q)\left\{F(P, i k)+M_{h}\left[u_{i}{ }^{h}(Q)\right]-L u_{i}{ }^{h}(Q)\right\} \\
& +\sum_{Q \in C_{n}} G_{0}{ }^{h}(P, Q) \cdot f(Q, i k), \quad i \geqq 0
\end{aligned}
$$

and $u_{0}^{k, h}(P):=0$

Associated with the problem (4.4) we define a discrete Green's function $G^{k, h}$ defined on $\bar{\Omega}_{h} \times \bar{\Omega}_{h} \times \mathbf{N}$ as follows:

$$
\begin{aligned}
& G^{k, h}(P, Q ; 0):=0 \\
& G^{k, h}(P, Q ; i):=\sum_{n=0}^{i}\left(\begin{array}{l}
i \\
n
\end{array}\right) k^{n} G_{n-1}^{h}(P, Q) \text {. for } i>0
\end{aligned}
$$

where the $G_{n}{ }^{n}, n \geqq 0$, are defined by (3.3). It is easy to show that $G^{k, h}$ acts as a re, producing singularity. To this end we observe the following result, whose proof is direct.

Theorem 4.5: $G^{k, h}$ satisfies

$$
\left.\begin{array}{lll}
\mathfrak{Q}_{k, h}^{F} G^{k, h}(P, Q ; i)=-h^{-2} \delta(P, Q) & \text { for } P \in \Omega_{h}, & i \in \mathbf{N}, \\
G^{k, h}(P, Q ; i)=i k \delta(P, Q) & \text { for } P \in \bar{C}_{h}, & i \in \mathbf{N}^{+} \\
G^{k, h}(P, Q ; 0)=0 & \text { for } P, Q \in \bar{\Omega}_{h} .
\end{array}\right\}
$$


The following representation theorem may be used to solve the nonhomogeneous initial-boundary value problem.

Theorem 4.6: Let $\left\{v_{i}^{h}\right\}_{i \in N}$ be defined on $\bar{\Omega}_{h}^{*}$ where $v_{0}{ }^{h} \equiv 0$. Then for $i \epsilon N$ and for $P \in \bar{\Omega}_{h}$

$$
\begin{aligned}
v_{i+1}(P)= & -h^{2} \sum_{j=0}^{i} \sum_{Q \in Q_{h}}\left[G^{k, h}(P, Q ; i+1-j)-G^{k, h}(\dot{P}, Q ; i-j)\right] \mathfrak{Q}_{k, h}^{F} v_{j+1}(Q) \\
& +k^{-1} \sum_{j=0}^{i} \sum_{Q \in C_{h}}\left[G^{k, h}(P, Q ; i+1-j)-G^{k, h}(P, Q ; i-j)\right] \\
& \times\left[v_{j+1}(Q)-v_{j}(Q)\right] .
\end{aligned}
$$

Proof: Let $w(P)$ denote the right-hand side of (4.8). For $P \in \bar{\Omega}_{h}$, we have

$$
\begin{aligned}
\mathfrak{Q}_{k, h}^{F} w(P)= & -h^{2} \sum_{j=0}^{i-1} \sum_{Q \in \Omega_{h}}\left[-h^{-2} \delta(P, Q)+h^{-2} \delta(P, Q)\right] \mathfrak{Q}_{k, h}^{F} v_{j+1}(Q) \\
& -h^{2} \sum_{Q \in \Omega_{h}}\left[-h^{-2} \delta(P, Q)-0\right] \mathfrak{L}_{k, h}^{F} v_{i+1}(Q) \\
& +k^{-1} \sum_{j=0}^{i-1} \sum_{Q \in C_{h}}\left[-h^{-2} \delta(P, Q)+h^{-2} \delta(P, Q)\right]\left[v_{j+1}(Q)-v_{j}(Q)\right] \\
& +k^{-1} \sum_{Q \in C_{n}}^{\longrightarrow}\left[-h^{-2} \delta(P, Q)-0\right]\left[v_{i+1}(Q)-v_{i}(Q)\right]
\end{aligned}
$$

Only the second term above fails to vanish, and it equals $\mathcal{Q}_{k, h}^{F} v_{i+1}(P)$.

If $P \in C_{h}$,

$$
\begin{aligned}
w(P)= & -h^{2} \sum_{j=0}^{i} \sum_{Q \in Q_{n}}[(i-j+1) k \delta(P, Q)-(i-j) k \delta(P, Q)] \dot{\mathfrak{Q}}_{k, h}^{F} v_{j+1}(Q) \\
& +k^{-1} \sum_{j=0}^{i} \sum_{Q \in C_{h}}[(i-j+1) k \delta(P, Q) \\
& -(i-j) k \delta(P, Q)]\left[v_{j+1}(Q)-v_{j}(Q)\right] \\
= & \sum_{j=0}^{i}\left[v_{j+1}(P)-v_{j}(P)\right]=v_{i+1}(P)-v_{0}(P)=v_{i+1}(P)
\end{aligned}
$$

The proof is completed by invoking Theorem 4.4 .

Formula (4.8) is simplified somewhat by using the identity

$$
G^{k, n}(P, Q ; i+1-j)-G^{k, n}(P, Q ; i-j)=\sum_{n=0}^{i-j}\left(\begin{array}{c}
i-j \\
n
\end{array}\right) k^{n+1} G_{n}{ }^{n}(P, Q)
$$

\section{Defining}

$$
\Gamma_{i+1, j}^{k, h}(P, Q):=\sum_{n=0}^{i-j}\left(\begin{array}{c}
i-j \\
n
\end{array}\right) k^{n} G_{n}{ }^{h}(P, Q)
$$

we may rewrite (4.8) in the form

$$
\begin{aligned}
v_{i+1}(P)= & -h^{2} k \sum_{j=0}^{i} \sum_{Q \in Q_{n}} \Gamma_{i+1, j}^{k . h}(P, Q) \mathfrak{Q}_{k, h}^{F} v_{j+1}(Q) \\
& +\sum_{j=0}^{i} \sum_{Q \in C_{n}} \Gamma_{i+1, j}^{k, h}(P, Q)\left[v_{j+1}(Q)-v_{j}(Q)\right]
\end{aligned}
$$




\section{อ. Error Bounds}

In this section we incorporate the usual error estimates which have been developed for elliptic partial differential equations, solutions represented in terms of fundamental singularities. $[2,3,16]$. In order to extend these estimates to the case of psendoparabolic equations we must first establish several lemmas:

Lemma 5.1: For each $i \geqq 0$, and for $0 \leqq j \leqq i, \Gamma_{i+1, j}^{k, h} \geqq 0$, where $\Gamma_{i+1, j}^{k, h}$ is defined in (4.10).

Proof: We know $G_{0}{ }^{h} \geqq 0$ by the Collatz maximum principal. Further, for $n \geqq 0$,

$$
G_{n+1}^{h}(P, Q)=-h^{2} \sum_{T \in \Omega_{n}} G_{0}{ }^{h}(P, T) a(T) G_{n}{ }^{n}(T, Q),
$$

, and since $a \leqq 0$, it follows by induction that $G_{n}{ }^{n} \geqq 0$ for all $n$. Hence, the desired result is immediate from (4.10)

Lemma 5.2: For $P \in \Omega_{h}, h^{2} \cdot \sum_{Q \in \Omega_{\Lambda}} G_{0}{ }^{h}(P, Q) \leqq K:=\left[\min _{Q \in \Omega_{h}} q(Q)\right]^{-1}$

Proof: As usual, let $C_{h}{ }^{*}$ denote all points of $\Omega_{h}$ which are neighbors of points in $C_{h}$, and for $Q \in \Omega_{h}$ let $N_{h}(Q)$ denote the set of neighbors of $Q$. Then for $P \in \Omega_{h}$,

$$
\mathbf{1}=-h^{2} \sum_{Q \in \Omega_{h}} G_{0}{ }^{h}(P, Q)[-q(Q)]+h^{2} \sum_{Q \in C^{*}} G_{0}{ }^{h}(P, Q) \cdot \operatorname{card}\left(N_{h}(Q) \cap C_{h}\right) .
$$

Since $G_{0}{ }^{h} \geqq 0$, we conclude

$$
1 \geqq h^{2} K^{-1} \sum_{Q \in \Omega_{h}} G_{0}{ }^{h}(P, Q) ;
$$

from which the desired result easily follows

Lenma 5.3: Let $i \geqq 0$ and let $0 \leqq j \leqq i$. Then for $P \in \Omega_{h}$,

$$
h^{2} \sum_{Q \in \Omega_{h}} \Gamma_{i+1, j}^{k, h}(P, Q) \leqq \dot{K}\left[i+k\|a\|_{\infty} K\right]^{i-j}
$$

. Proof: For $n \geqq 0$ and $P \in \Omega_{h}$,

$$
\begin{aligned}
& h^{2} \sum_{Q \in \Omega_{n}} G_{n+1}^{h}(P, Q)=h^{2} \sum_{Q \in \Omega_{n}}\left[-h^{2} \sum_{T \in \Omega_{n}} G_{0}{ }^{\dot{h}}(P, T) a(T) G_{n}{ }^{h}(T, Q)\right] \\
& =h^{2} \sum_{T \in \Omega_{n}}[-a(T)] G_{0}{ }^{h}(P, T) h^{2}\left[\sum_{Q \in \Omega_{h}^{\prime}} \dot{G}_{n}{ }^{h}(T, Q)\right] \\
& \leqq\|a\|_{\infty}\left[\max _{T \in \Omega_{n}} h^{2} \sum_{0 \in \Omega_{\mathrm{h}}} G_{n}{ }^{h}(T, Q)\right]\left[\begin{array}{c}
h^{2} \sum_{T \in \Omega_{n}} G_{0}{ }^{h}(P ; T) \\
.
\end{array}\right]
\end{aligned}
$$

By induction, it follows that for $n \geqq 0$ and $P \in \Omega_{h}$,

$$
h^{2} \sum_{Q \in \Omega_{n}} G_{n}{ }^{n}(P, Q) \leqq\|\dot{a}\|_{\infty}^{n} K^{n+1}
$$

Inequality (5.1) easily follows now from (4.10)

Finally, we bound the error $\varepsilon_{i}^{k, h}(P):=u(P, i k)-u_{i}^{k, h}(P)$, where $u_{i}^{k, h}$ is a solution of (4.4). We have $\varepsilon_{0}{ }^{k, h} \equiv 0$ and $\varepsilon_{i}^{k, h}(P)=0$ for $P \in C_{n}$. For $i \geqq 0$ and $P \in \Omega_{h}$, we apply (4.11) to obtain

$$
\begin{aligned}
\varepsilon_{i+1}^{k, h}(P)= & -h^{2} k \sum_{j=0}^{i} \sum_{Q \in \Omega_{h}} \Gamma_{i+1, j}^{k, h}(P, Q) \mathfrak{Q}_{k, h}^{F} \varepsilon_{j+1}(Q) \\
= & -h^{2} k \sum_{j=0}^{i} \sum_{Q \in \Omega_{h}} \Gamma_{i+1, j}^{k, h}(P, Q)\left\{M_{h}[u(Q,(j+1) k)-u(Q, j k)]\right. \\
\therefore \quad & -M u(Q, j k)\} .
\end{aligned}
$$


So applying (5.1) we find

$$
\left|\varepsilon_{i+1}^{k, h}(P)\right| \leqq x_{i+1} K k^{i} \sum_{j=0}^{i}\left[1+k\|a\|_{\infty} K\right]^{i-j} \leqq \varkappa_{j+1} K(i+1) k\left[1+k\|a\|_{\infty} K\right]^{i},
$$

where

$$
\varkappa_{i+1}:=\max _{0 \leqq j \leqq i} \operatorname{mix}_{Q \in \Omega_{n}}\left|M_{h}[u(Q,(j+1) k)-u(Q, j k)]-M u(Q, j k)\right| .
$$

Our error bound now follows from (5.2) under typical continuity assumptions on $u$.

Theorẹin 5.4: Let u be simultaneously $C^{4}$ on $\Omega$ and $C^{2}$ on $[0, T]$. Then therè exists a constant $x$ independent of $k$ and $h$, such that for $P \in \Omega_{h}$ and $0 \leqq i \leqq m+1 \leqq[T / k]$,

$$
\left|\varepsilon_{i}{ }^{k, h}(P)\right| \leqq x K T\left[1+\dot{k}\|\dot{a}\|_{\infty} K\right]^{m} k h^{2}
$$

\section{Concluding Remarks}

'It is not necessary that $\Omega$ be a rectangle; indeed, we may consider irregular regions $\Omega$, providing that $\dot{S}$ is sufficiently smooth. We ask that $\dot{\Omega}$ be such that our Green's identities hold. As before, we place a rectangular grid of equal spacing over $\Omega$. 'I'he boundáry points, which we shall designate as points of $C_{h}$; are the intersections of $\dot{\Omega}$ with the grid lines. Next" we designate an "inner boundary" $C_{n}{ }^{*}$, as the set of points which are nearest neighbörs to points of $C_{n}$. The remaining points of $\Omega$ which coincide "with grid points are "interior points" and their collection is designated by $\Omega_{h}$. The closure of $\Omega_{h}$ is $\bar{\Omega}_{h}:=\Omega_{h}+C_{h}+C_{h}^{*}$.

For the most part, we may, proceed as before by replacing in our definitions and - formulae $\Omega_{h}$ by $\Omega_{h}+C_{h}{ }^{*}$. Some concern must be taken, however, when dealing with the Laplacian on points of $C_{h}{ }^{*}$. For this case, we choose an interpolated Laplacian [3]

$$
\begin{aligned}
\triangle \operatorname{si}^{\prime} u:= & h^{-2}\left\{\frac{u\left(x+\alpha_{1} h, y\right)}{\left(\alpha_{1}+\alpha_{2}\right) \alpha_{1}}+\frac{u\left(x-\alpha_{2} h, y\right)}{\left(\alpha_{1}+\alpha_{2}\right) \alpha_{2}}+\frac{u\left(x, y+\beta_{1} h\right)}{\left(\beta_{1}+\beta_{2}\right) \beta_{1}}\right. \\
& \left.+\frac{u\left(x, y-\beta_{1} h\right)}{\left(\beta_{1}+\beta_{2}\right) \beta_{2}}-\left(\frac{1}{\alpha_{1} \alpha_{2}}+\frac{1}{\beta_{1} \beta_{2}}\right) u(x, y)\right\},
\end{aligned}
$$

'where $0<\alpha_{1}, \alpha_{2}, \beta_{1}, \beta_{2} \leqq 1$. Not all of these parameters will be strictly less than one, since the distances $\alpha_{i} h, \beta_{i} h(i \doteq 1,2)$ where $\alpha_{i}$, or $\beta_{i}<1$ are measured from a point on $C_{h}{ }^{*}$ to a neighbour or $C_{h}$, and $h$ is chosen small enough so that $\Omega_{h}$ is simply connected.

To verify Lemma (5.2) for the present case, we introduce the function

$$
Z(P):=\left\{\begin{array}{l}
1, P \in \Omega_{h}+C_{h}^{*}, \\
0, P \in C_{h} .
\end{array}\right.
$$

Proceeding as before we employ a discrete Green's identity $[2,3,16]$ associated with the finite-difference operator

$$
M_{h}[U]:=\left(\triangle_{h}-q\right) U
$$

Hence, for irregular regions we have

$$
\dot{U}(P) \doteq-h^{2} \sum_{Q \in \Omega_{n}+C_{n}{ }^{*}} M_{h}[U](Q) G_{0}(\dot{P}, Q)+\sum_{Q \in C_{n}} G_{0}(P, Q) U(Q)
$$


Now inserting $Z(P)$ for $U(P)$ in (6.3) yields ,

$$
1=h_{Q \in \Omega_{h}+C_{h^{*}}} \sum_{Q}(Q) G_{0}(P, Q)-h^{2} \sum_{Q \in C_{h}^{*}}\left(\triangle_{h} Z\right)(Q) G_{0}(P, Q) \text { for } P \in \Omega_{h}+C_{h}{ }^{*}
$$

A short computation with the interpolated Laplacian (6.1) verifies that $\left(\triangle_{h} Z\right)(Q)<0$ for $Q \in C_{h}{ }^{*}$; hence, as before, we have the inequality of Lemma (5.2), namely

$$
1 \geqq h_{Q \in Q_{\mathrm{n}}+C_{\mathrm{n}}^{*}} q(Q) G_{0}(P, Q) .
$$

It is now clear that the formula for the error estimate (5.3) may be extended to the present case when we define

$$
K:=\max _{Q \in \Omega_{n}+C_{n}^{*}}[q(Q)]^{-1} \text { and }\|a\|_{\infty}:=\sup _{Q \in \Omega_{n}+C_{n}^{*}}[a(Q)]
$$

\section{REFERENCES}

[1] BarEn BLAT, G., Zheltov, I., and I. Kocriva: Basic concepts in the theory of seepage of homogeneous fluids in fissured rock. J. Appl. Math. Mech. 24 (1960), 1286-1303.

[2] Bramble, I., and B. Hubbard: A priori bounds on the discretization error in the numerical solution of the Dirichlet problem. Contrib. Diff. Equat. 1 (1963), 229-252.

[3] Collatz, L.: The Numerical Treatment of 'Differential Equations'(3rd ed.). Springer-Verlag: Berlin 1960.

[4] Colton, D. L.: Integral operators and the first initial boundary value problem for pseudoparabolic equations with analytic coefficients. J. Diff. Equat. 13 (1973), 506-522.

[5] Colton, D. L.: On the analytic theory of pseudoparabolic equations. Quart. J. Math. 23 (1972), $179-192$.

[6] Cotrant, D. L., and D. Hilbert: Methods of Mathematical Physics (Vol. II). Wiley: New York' 1962.

[7] GrLbert, R: P.: A Lewy-Type' reflection principle for pseudoparabolic equations. Journal of Diff. Equat. 37 (1980), 261-284.

[8] Gilbert, R.P., and G.C. HsiaO: Constructive function theoretic methods for higher order pseudoparabolic equations. In: Lecture Notes in Math. 561. Springer: Berlin 1976.

[9] Showalter, R. E.: Well-posed problems for a partial differential equation of order $2 m+1$. SIAM J. Math. Anal. 1 (1970), 214-231.

[10] Showalter, R. E.: Partial differential equations of Sobolev-Calpern type. Pacific J. Math. 31 (1969), $789-794$.

[11] Showalter, R. E., and T. W. Tiva: Pseudoparabolic partial differential equations. SIAM/ J. Math. Anal. 1 (1970), 1 - 26.

[12] TAYLOR, D. W.: Research on Consolidation of Clays. MIT Press: Cambridge 1942.

[13] Trng, 'T. W.: Parabolic and pseudoparabolic parțial differential equations. J: Math. Soc. Japan 21 (1969), 440-453.

[14] 'Ting, T. W.: Certain non-steady flows of second order fluids. Arch. Rat. Mech. Anal. 14 (1963), $1-26$.

[15] Walter, W:: Differential and Integral Inequalities. Ergeb. Math. Grenzgeb. Bd. ј5. Springer-Verlag: Berlin 1970.

[16] Wendeaxd, W.: Elliptic Systems in the Plane. Pitman-Verlag: London 1979.

Manuskripteingang: 26.2.1982

\section{VERFASSER:}

Prof. Dr. Robert P. Gilbert and Dr. Leroy R. Lundin

Applied Mathematics Institute of the University of Delaware

Rees Hall, 5 West Main Street

Newark, Delaware 19711, USA 